\title{
Nonsuppressible HIV-1 viremia: a reflection of how the reservoir persists
}

\author{
Janet D. Siliciano ${ }^{1}$ and Robert F. Siliciano ${ }^{1,2}$ \\ 'Department of Medicine, Johns Hopkins University School of Medicine, Baltimore, Maryland, USA. ${ }^{2}$ Howard Hughes Medical Institute, Baltimore, Maryland, USA.
}

\begin{abstract}
Antiretroviral therapy (ART) generally reduces plasma HIV to undetectable levels, although virus persists in latently infected CD4+ $T$ cells. In some individuals, viremia remains detectable despite adherence to ART and the absence of drug resistance mutations. In this issue of the $J C I$, Halvas et al. describe HIV RNA sequences from plasma of 8 donors with persistent viremia. Residual viremia was dominated by identical HIV-1 RNA sequences that remained relatively constant over 4 years. Plasma virus matched replication-competent virus cultured from $\mathrm{CD4}^{+} \mathrm{T}$ cells. Integration site analysis confirmed the presence of large clones of infected cells. These results indicate that nonsuppressible viremia can be due to expanded clones of infected $\mathrm{CD}^{+} \mathrm{T}$ cells carrying replication-competent virus. The individuals described here represent extreme examples of a phenomenon that is seen in all infected individuals and that is a major barrier to curing HIV infection, the in vivo proliferation of latently infected cells.
\end{abstract}

\section{Nonsuppressible HIV-1 viremia}

Rare is the paper that provides insight into a fundamental scientific issue while also solving a long-standing clinical dilemma. In this issue of the JCI, Halvas et al. (1) explored the clinical dilemma that some HIV-1-infected individuals on optimal antiretroviral drug regimens continue to have detectable viremia. Since the introduction of effective combination antiretroviral therapy (ART) in 1997 (2, 3), the aim of treatment has been to suppress plasma virus levels to below the limit of detection. With good adherence, suppressing detectable viremia is generally achievable and allows near-normal life expectancies (4). However, due to the persistence of a stable reservoir of latently infected cells (5-8), viremia rebounds within weeks if ART is interrupted (9), and progression toward AIDS resumes. Therefore, sustained sup- pression of detectable viremia is the goal of treatment. When viremia remains or becomes detectable in people on ART, clinicians suspect problems with adherence or, less commonly, mutations conferring resistance to drugs in the regimen or issues with drug metabolism. Alternative regimens are often recommended. Rarely, viremia remains detectable in individuals who are adherent, have no resistance mutations, and have drug levels in the therapeutic range. This scenario presents a challenge for clinical management. Halvas et al. provide one fascinating explanation for this phenomenon involving the clonal expansion of latently infected cells.

To understand nonsuppressible HIV-1 viremia, it is useful to consider how viremia is measured. Clinical assays for plasma HIV-1 RNA typically use some form of quantitative RT-PCR to measure genomic

Related Article: p. 5847

Conflict of interest: RFS is an inventor on a patent application for the intact proviral DNA assay (IPDA) filed by Johns Hopkins University and licensed by AccelevirDx: "Novel assay for accurately measuring and characterizing the HIV-1 latent reservoir" (patent no. 62/152,436)

Copyright: @ 2020, American Society for Clinical Investigation.

Reference information: J Clin Invest. 2020;130(11):5665-5667. https://doi.org/10.1172/JCl141497.

viral RNA, 2 copies of which are present in each virion (10). In untreated individuals, typical values are in the range of 10,000 to 100,000 copies of HIV-1 RNA per mL of plasma. Following initiation of ART, viremia falls in biphasic fashion to below the limit of detection for these assays (20-50 copies/mL) within a few months $(2,3)$. Much evidence suggests that ART regimens cause a complete block in new infection of susceptible cells (reviewed in ref. 11), and therefore this rapid decay in viremia reflects the turnover of cells that were infected at the time treatment was started (2). Interestingly, although ART suppresses viremia to below the limit of detection of clinical assays, sensitive research assays can still detect virus in the plasma (12, 13). It appears that ART simply reduces viremia to a new steady-state level that is slightly below the limit of detection of clinical assays (Figure 1A). This trace level of residual viremia is often in the range of 1-3 copies/mL $(13,14)$. The residual viremia appears to represent virus that originates from the latent reservoir rather than ongoing cycles of replication because it cannot be further reduced by intensifying treatment $(15,16)$.

\section{The latent reservoir}

Given that all treated individuals have some level of viremia derived from the latent reservoir, it is logical to assume that in rare individuals who have an unusually high frequency of latently infected cells, the steady-state level of plasma virus derived from the reservoir may be high enough to be detected with clinical assays. Unfortunately, measuring the frequency of latently infected cells is complicated by several factors, including the high frequency of defective viral genomes (17), and is not yet part of clinical management. The original viral outgrowth assay (6) detects replication-competent viruses but misses many viruses in the reservoir that are not activated in this assay $(18,19)$. Standard DNA PCR assays mainly detect 
A

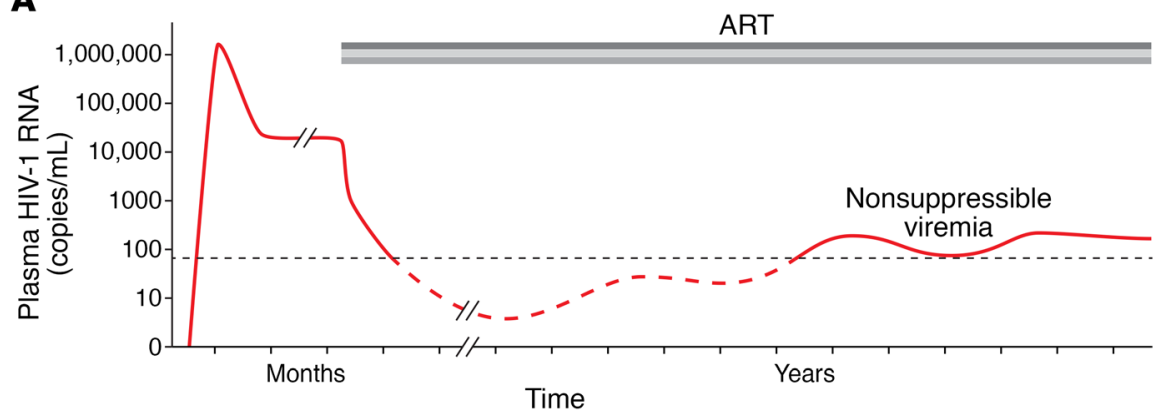

B

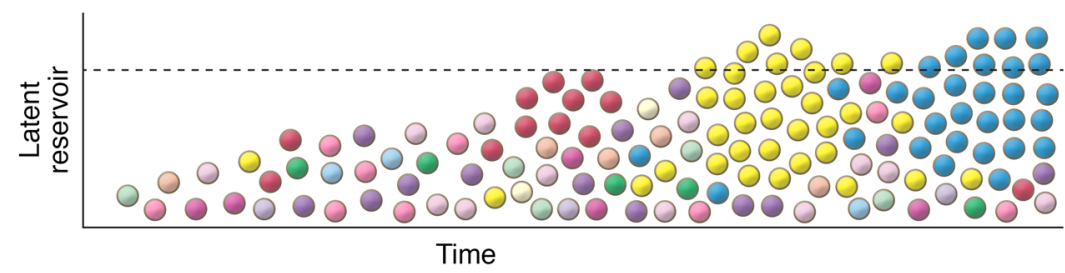

Figure 1. Nonsuppressible viremia can result from large clones of infected cells. (A) Plasma virus levels before and following initiation of antiretroviral therapy (ART). ART, represented as 3 gray bars to show a combination therapy regimen, generally blocks new infection of susceptible cells and reduces viremia to below the limit of detection of clinical assays (dotted black line). However, low-level viremia persists (dashed red line), representing virus release from cells in the latent reservoir that have become activated. (B) $\mathrm{CD} 4^{+} \mathrm{T}$ cells comprise the latent reservoir, with colors representing different clonal lineages. The size and composition of the reservoir are generally such that the level of virus released from reservoir cells is below the limit of detection of clinical assays (dotted line). However, in some individuals, clones can expand to a very large size (yellow and blue cells) and release a sufficient amount of virus to produce detectable viremia. This viremia cannot be suppressed by ART, which continues to block new infection of susceptible cells but not virus release from previously infected cells.

defective proviruses and are not useful (17). Newer assays such as the intact proviral DNA assay (IPDA) may eventually allow accurate measurement of reservoir size in the clinic (20). Using older reservoir assays, Halvas et al. found that some but not all individuals with nonsuppressible viremia had a high frequency of latently infected cells. However, rather than focusing on reservoir size, the researchers addressed another aspect of the problem that explains how the reservoir expands and persists.

The reservoir consists of resting $\mathrm{CD} 4^{+}$ $\mathrm{T}$ cells, each carrying a stably integrated viral genome that is transcriptionally silent as long as the cell remains in a resting state (21). The absence of viral gene expression allows latently infected cells to avoid elimination by immune mechanisms. In recent years, it has become clear that cells comprising the reservoir can undergo extensive proliferation in vivo, generating large clones of $\mathrm{CD} 4^{+} \mathrm{T}$ cells that carry identical proviruses integrated into an identical position in the human genome. The first evidence for the proliferation of latently infected cells came from early studies examining the residual viremia in treated individuals who had plasma virus levels below the limit of detection of clinical assays $(22,23)$. These studies showed that the residual viremia is often dominated by identical viral sequences likely derived from clones of infected cells. Subsequent studies used integration site analysis to provide definitive proof for the in vivo proliferation of infected cells $(24,25)$. Viral outgrowth assays were then used to show that $\mathrm{CD}^{+} \mathrm{T}$ cells carrying replication-competent viral genomes could proliferate in vivo $(18,26-28)$. It is now clear that the latent reservoir is dominated by large clones of infected cells. Interestingly, these clones do not continually expand. Rather, they appear to wax and wane on a time scale of month to years (29), such that the total size of the reservoir remains roughly constant.

Halvas and colleagues asked whether cases of nonsuppressible viremia could be attributed to the presence of large clones of infected cells that release identical viruses that can be detected in the plasma. They studied 8 infected individuals who had persistently detectable viremia (median value, 87 copies $/ \mathrm{mL}$ ) for an average of 4.1 years. In all 8 individuals, sequencing residual viremia revealed the presence of identical sequences, which comprised an average of $58 \%$ of the sequences obtained. In infected individuals who started ART during the chronic phase of infection, rapid viral evolution generates a vast diversity of viral sequences, and thus the presence of a large number of identical sequences is unexpected and can only be explained by extensive proliferation of a clone of infected cells, which would copy the viral genome without error into all of the progeny cells. If the clone is sufficiently large, virus production by only a small fraction of the cells comprising the clone could give rise to levels of viremia in the detectable range (Figure 1B). Importantly, Halvas et al. provided definitive proof that at least in some cases the identical sequences were truly representative of infected cell clones by showing that multiple cells carrying these identical viral sequences had exactly the same proviral integration sites. They also proved that at least in some cases the viruses were replication competent as assessed by in vitro replication in a viral outgrowth assay.

\section{Clinical implications}

The study by Halvas et al. has important implications for clinical management and for the search for a cure. It now appears that in some cases nonsuppressible viremia can be attributed to large clones of infected cells that release virus into the plasma. If other causes of persistent viremia are ruled out, then this form of viremia need not necessitate a change in treatment. This phenomenon does highlight a major challenge we face in the search for a cure, namely the fact the latently infected cells that represent a major barrier to cure can proliferate in vivo. Understanding what causes the proliferation is crucial. Previous studies have suggested that proviral integration into certain cellular genes involved in cell survival or proliferation might drive the clonal expansion of infected cells through a form of promoter insertion mutagenesis $(24,25)$. The integration sites identified in Halvas et al. are in genes that do not have an obvious 
role in proliferation, and it is likely that the observed proliferation is driven by physiologic stimuli. Unfortunately, it will be difficult to block this form of proliferation without compromising normal $\mathrm{T}$ cell function. Direct targeting of latently infected cells may be necessary. This direct targeting will likely require reversal of latency so that immune effector mechanisms can recognize and eliminate infected cells. A recent study has indicated that expanded $\mathrm{CD}^{+}$ $\mathrm{T}$ cell clones carrying replication-competent virus are present in some people who control HIV infection without ART and that $\mathrm{CD} 8^{+}$cytolytic T lymphocytes can suppress replication of virus produced by these clones (30). At least some cells comprising the expanded cellular clones described here (1) produced virus, but it is clear that latently infected cells can proliferate without releasing virus $(18,19)$. It is possible that the nonsuppressible viremia described here represents virus production from only a small fraction of the cells comprising the clones. To achieve a cure, we may need to find ways to reverse latency and eliminate most or all latently infected cells.

Address correspondence to: Janet D. Siliciano, Department of Medicine, Johns Hopkins University School of Medicine, 879 MRB, 733 N. Broadway, Baltimore, Maryland 21205, USA. Phone: 410.955.7757; Email:jsilicia@jhmi.edu.

1. Halvas EK, et al. HIV-1 viremia not suppressible by antiretroviral therapy can originate from large $\mathrm{T}$ cell clones producing infectious virus. JClin Invest. 2020;130(11):5847-5857.

2. Perelson AS, et al. Decay characteristics of HIV-1-infected compartments during combination therapy. Nature. 1997;387(6629):188-191.

3. Gulick RM, et al. Treatment with indinavir, zidovudine, and lamivudine in adults with human immunodeficiency virus infection and prior antiretroviral therapy. $N$ Engl J Med. 1997;337(11):734-739.
4. Nakagawa F, May M, Phillips A. Life expectancy living with HIV: recent estimates and future implications. Curr Opin Infect Dis. 2013;26(1):17-25.

5. Chun TW, Finzi D, Margolick J, Chadwick $\mathrm{K}$, Schwartz D, Siliciano RF. In vivo fate of HIV-1-infected T cells: quantitative analysis of the transition to stable latency. Nat Med. 1995;1(12):1284-1290.

6. Finzi D, et al. Identification of a reservoir for HIV-1 in patients on highly active antiretrovira therapy. Science. 1997;278(5341):1295-1300.

7. Wong JK, et al. Recovery of replication-competent HIV despite prolonged suppression of plasma viremia. Science. 1997;278(5341):1291-1295.

8. Chun TW, et al. Presence of an inducible HIV-1 latent reservoir during highly active antiretroviral therapy. Proc Natl Acad Sci US A. 1997;94(24):13193-13197.

9. Davey RT, et al. HIV-1 and T cell dynamics after interruption of highly active antiretroviral therapy (HAART) in patients with a history of sustained viral suppression. Proc Natl Acad Sci US A. 1999;96(26):15109-15114.

10. Piatak M, et al. High levels of HIV-1 in plasma during all stages of infection determined by competitive PCR. Science. 1993;259(5102):1749-1754.

11. Siliciano JD, Siliciano RF. Recent trends in HIV-1 drug resistance. Curr Opin Virol. 2013;3(5):487-494.

12. Dornadula G, et al. Residual HIV-1 RNA in blood plasma of patients taking suppressive highly active antiretroviral therapy. JAMA. 1999;282(17):1627-1632.

13. Maldarelli F, et al. ART suppresses plasma HIV-1 RNA to a stable set point predicted by pretherapy viremia. PLoS Pathog. 2007;3(4):e46

14. Palmer S, et al. New real-time reverse transcriptase-initiated PCR assay with single-copy sensitivity for human immunodeficiency virus type 1 RNA in plasma. J Clin Microbiol. 2003;41(10):4531-4536.

15. Dinoso JB, et al. Treatment intensification does not reduce residual HIV-1 viremia in patients on highly active antiretroviral therapy. Proc Natl Acad Sci U S A. 2009;106(23):9403-9408

16. Gandhi RT, et al. The effect of raltegravir intensification on low-level residual viremia in HIV-infected patients on antiretroviral therapy: a randomized controlled trial. PLoS Med. 2010;7(8):e1000321.

17. Ho YC, et al. Replication-competent noninduced proviruses in the latent reservoir increase barrier to HIV-1 cure. Cell. 2013;155(3):540-551.

18. Hosmane NN, et al. Proliferation of latently infected $\mathrm{CD} 4^{+} \mathrm{T}$ cells carrying replicationcompetent HIV-1: Potential role in latent reservoir dynamics. J Exp Med. 2017;214(4):959-972.

19. Kwon KJ, et al. Different human resting memory $\mathrm{CD}^{+} \mathrm{T}$ cell subsets show similar low inducibility of latent HIV-1 proviruses. Sci Transl Med. 2020;12(528):eaax6795.

20. Bruner KM, et al. A quantitative approach for measuring the reservoir of latent HIV-1 proviruses. Nature. 2019;566(7742):120-125.

21. Siliciano RF, Greene WC. HIV latency. Cold Spring Harb Perspect Med. 2011;1(1):a007096.

22. Tobin NH, et al. Evidence that low-level viremias during effective highly active antiretroviral therapy result from two processes: expression of archival virus and replication of virus. J Virol. 2005;79(15):9625-9634.

23. Bailey JR, et al. Residual human immunodeficiency virus type 1 viremia in some patients on antiretroviral therapy is dominated by a small number of invariant clones rarely found in circulating $\mathrm{CD}^{+} \mathrm{T}$ cells. $J$ Virol. 2006;80(13):6441-6457.

24. Maldarelli F, et al. HIV latency. Specific HIV integration sites are linked to clonal expansion and persistence of infected cells. Science. 2014;345(6193):179-183.

25. Wagner TA, et al. HIV latency. Proliferation of cells with HIV integrated into cancer genes contributes to persistent infection. Science. 2014;345(6196):570-573.

26. Simonetti FR, et al. Clonally expanded CD $4^{+} \mathrm{T}$ cells can produce infectious HIV-1 in vivo. Proc Natl Acad Sci U S A. 2016;113(7):1883-1888.

27. Bui JK, et al. Ex vivo activation of $\mathrm{CD} 4^{+} \mathrm{T}$-cells from donors on suppressive ART can lead to sustained production of infectious HIV-1 from a subset of infected cells. PLoS Pathog 2017;13(2):e1006230.

28. Lorenzi JC, et al. Paired quantitative and qualitative assessment of the replication-competent HIV-1 reservoir and comparison with integrated proviral DNA. Proc Natl Acad Sci U S A 2016;113(49):E7908-E7916.

29. Wang Z, et al. Expanded cellular clones carrying replication-competent HIV-1 persist, wax, and wane. Proc Natl Acad Sci U S A. 2018;115(11):E2575-E2584.

30. Veenhuis RT, et al. Long-term remission despite clonal expansion of replication-competent HIV-1 isolates. JCI Insight. 2018;3(18):122795. 